\title{
Estimation and Control in Semiconductor Etch: Practice and Possibilities
}

\author{
John V. Ringwood, Senior Member, IEEE, Shane Lynn, Student Member, IEEE, Giorgio Bacelli, Beibei Ma, \\ Emanuele Ragnoli, and Sean McLoone, Senior Member, IEEE
}

\begin{abstract}
Semiconductor wafer etching is, to a large extent, an open-loop process with little direct feedback control. Most silicon chip manufacturers rely on the rigorous adherence to a "recipe" for the various etch processes, which have been built up based on considerable historical experience. However, residue buildup and difficulties in achieving consistent preventative maintenance operations lead to drifts and step changes in process characteristics. This paper examines the particular technical difficulties encountered in achieving consistency in the etching of semiconductor wafers and documents the range of estimation and control techniques currently available to address these difficulties. An important feature of such an assessment is the range of measurement options available if closed-loop control is to be achieved.
\end{abstract}

Index Terms-Closed-loop control, plasma etch, run-to-run control, semiconductor wafer, state estimation, virtual metrology.

\section{INTRODUCTION}

W ITH the continuing drive towards smaller feature sizes [1], there are increased pressures on tolerances in semiconductor wafer processing. As the unit value of wafers increases with these dimension decreases, it is imperative that better quality control be achieved if yield rates are to be maintained. Closed-loop control, and related technologies, are traditionally employed to reduce process variance, with the ability to tightly regulate around process setpoints directly relating to yield. Many of the control issues in semiconductor manufacturing are covered in [2]-[6].

Semiconductor manufacturing processes, such as plasma etch, are highly complex processes, and the minute feature sizes also make etch variable measurement difficult, with measurement feedback a prerequisite for control. In addition, attempts to perform in-situ measurement can disturb the etch process, which is largely run according to a very specific open-loop recipe. Optical and electrical measurement of the wafer provide downstream measurement, which, though not usable for real-time control, could be used in a run-to-run control strategy.

In addition to measurement and control, two associated (and related) areas that can assist in quality improvement are mathematical modelling and virtual metrology. Mathematical modelling involves the determination of a set of mathematical equa-

Manuscript received March 05, 2008; revised August 09, 2009. First published December 22, 2009; current version published February 03, 2010. This work was supported by Intel (Ireland) Ltd., Enterprise Ireland, and the Irish Research Council for Science, Engineering and Technology.

The authors are with the Department of Electronic Engineering, National University of Ireland, Maynooth, Ireland (e-mail: john.ringwood@eeng.nuim.ie).

Digital Object Identifier 10.1109/TSM.2009.2039250 tions to describe the behavior of a process. Such mathematical models are usually embedded in virtual metrology algorithms and also form the basis for model-based control strategies. Virtual metrology (VM) models the relationship between desired unmeasurable variables and other measurable quantities. The model is then employed to "reconstruct" the variables, which are not directly measurable.

This paper is focused on the state-of-the-art in the control and measurement sciences as applied to the semiconductor etch process. It follows the excellent review of Edgar et al. [7] (in 2000) and the November 2007 issue of this TRANSACTIONS, which has a Special Section on Advanced Process Control [8]. This paper attempts to present a broad picture of the possibilities in etch control and estimation and presents a sample of the recent literature for illustration. More comprehensive literature listings are contained in [9]-[11].

\section{Mathematical Models}

The etch process is a multivariable (interacting), nonlinear, distributed parameter process with a significant spread of dynamical time constants. As such, some level of simplification is inevitable if models are to be tractable and computable. Plasma/ etch models can be considered at various levels.

- Particle-in-cell (PIC) models [12] model the spatial variation in the plasma and, in general, respect the very fastest process dynamics. They are computationally intensive due to the need to compute electric/magnetic fields at mesh points in three dimensions, integration of the equations of motion, and interpolation of the fields to the particle locations.

- Bulk plasma models, e.g., [13]-[15], ignore the spatial dimension and focus on the representation of the physical process as a lumped parameter system. While these models are computationally simpler than PIC models, they have no ability to resolve spatial variations, but single point modelled outputs usually focus on specific areas of interest, e.g., ion flux at the wafer surface.

- Black-box models, e.g., [16] and [17], largely ignore the underlying physical process and are parameterized from a process behavioral point of view. Measured process inputs and outputs are used to produce a mathematical formula relating outputs to inputs. While black-box models can be used to derive relationships between spatially distributed measurements, they are normally used for bulk or singlepoint measurements.

Due to an incomplete understanding of plasma physics, it is unlikely that a complete physical plasma model will be achieved. In addition, for some applications, such as model-based control 
and state estimation, models must have the facility to be interrogated in real time. For run-to-run control, computational speed is not so crucial. In general, PIC models [18] are less useful for control due to their computational complexity and the provision of information not crucial to the control problem. In addition, the dynamics of the kinetic reactions, which are accurately modelled by the PIC models, are very fast compared to the dominant (slow dynamics) in etch control loops, which arise primarily from actuator (e.g., throttle valve, gas flow) and sensor dynamics.

At the other end of the spectrum, black-box models [19], including artificial neural networks (ANNs) [17], require the minimum of process knowledge but are only valid for the range of data used to train the models and care needs to be taken in the specification of a parsimonious model structure. Bulk plasma models, based on physical principles, are frequently employed in etch control and can be tuned using process data. For example, a control-oriented plasma model is developed in [20], where a static, multivariable nonlinearity is used to model the plasma, with the (largely scalar) dynamics resulting from sensors and actuators.

Equivalent circuit models are also useful in plasma/etch modelling, particularly where virtual instrumentation relies on impedance measurements [21], and can also be useful in providing corrections to raw radio-frequency (RF) measurements [22].

The challenges and progress in modelling the spatial variation in plasma etch processes are articulated well in Yang et al. [23].

\section{Measurement and Virtual Metrology}

Measurement is vital for process monitoring, diagnosis, and control. Various measurement regimes are possible, including real-time local measurement (e.g., Langmuir probe, optical emission spectra (OES), plasma impedance monitor (PIM), etc.), virtual metrology (to synthesize, in real time, important variables that are not directly measurable), and downstream (delayed) metrology (electrical and optical). The availability of key process variable measurements in real time is crucial to accurate diagnosis and control of plasma etch processes.

\section{A. Diagnostic Measurements}

Some measurement devices can provide actual measurement of plasma or etch variables, e.g., the Langmuir probe provides direct measurement of ion flux, while laser inferometry directly measures etch depth. However, the in-situ use of such measurement devices in a production environment can be problematic. Other devices, such as OES and PIMs [24], provide a more indirect measurement of the process variables and can, at least, be used to diagnose the "health" of the process if normal working measurement profiles are known. However, they can also be incorporated in VM strategies to give an indirect measurement of the process variables, e.g., [25].

\section{B. Virtual Metrology}

Virtual metrology attempts to provide estimates of quantities that are not directly measurable, based on associated measurements and mathematical models relating the measured and unmeasured quantities. The essential principle of VM, in a semi-

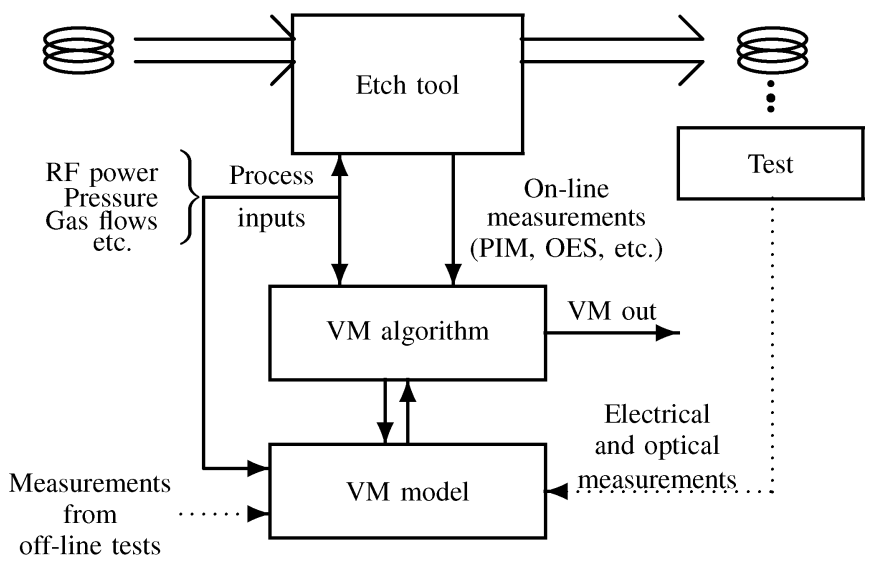

Fig. 1. Virtual metrology principle.

conductor etch setting, is shown in Fig. 1. Known in control parlance as state estimation (dating back to the pioneering work of Kalman [26]), VM can, for some model types (i.e., linear state-space models), provide a predictor/corrector-type structure, as is the case for the Kalman filter [26]

$$
\begin{array}{ll}
\text { Predictor : } & \hat{x}_{k+1 \mid k}=A \hat{x}_{k \mid k}+B u_{k} \\
\text { Corrector : } & \hat{x}_{k+1 \mid k+1}=\hat{x}_{k+1 \mid k}+K_{k} \tilde{y}_{k+1}
\end{array}
$$

where $\hat{x}_{k+1 \mid k}$ is the estimate of the state $x$ using the "open-loop" model $(A, B), u$ is the causal input from which $x$ is derived (e.g., process inputs), $\tilde{y}=y-C \hat{x}$ is the error in the model prediction of a measured variable $y(=C x)$, and $K$ is the Kalman gain. For the linear $(A, B, C)$ model, $K$ can be determined using an analytical procedure. In contrast to the Kalman filter, most estimation of plasma etch variables is done without a correction term, relying exclusively on the fidelity of the "predictor" model and therefore sensitive to modelling errors and disturbances between $u$ and $x$.

In general, it is desirable to estimate etch variables, such as etch rate, as regularly spaced samples in time. Endpoint detection is a somewhat special case of virtual metrology, since it focuses more on event, rather than continuous variable, estimation. By way of example, the paper by Lynn et al. [27] provides an example of a range of both statistical and neural network modelling paradigms applied for VM to tool variables, with the use of stepwise regression for variable selection.

1) Statistical Analysis: This section documents techniques that use some element of statistical analysis for virtual metrology. Many of the techniques relate to data mining, and some are based on dimensionality reduction [e.g., using principal component analysis (PCA)] and data preprocessing, including pattern analysis. A number of techniques also build mathematical models relating available measurements to key (unmeasurable) etch variables [e.g., partial least squares (PLS)].

The main techniques employed in this category are PCA and PLS. PCA [28] is an unsupervised technique that allows an array of measurements to be concisely described by a reduced number of "principal components," where an attempt is made to explain the majority of the variance in the original measurements using the minimum number of components. The derived principal components are mutually orthogonal and are ordered in 
terms of amount of variance explained in the original data. Typically, PCA is used to distill information distributed across a number of (potentially correlated) measurements into a smaller number of independent components.

An alternative to PCA, independent component analysis (ICA) [29], can also be used to separate independent signal streams, where the new data basis is formed by a set of independent vectors, rather than orthogonal vectors, as in the case of PCA. Though ICA has found considerable application in areas such as blind source separation [30], the application to semiconductor etch has been limited [31].

Supervised statistical techniques such as PLS and a supervised derivative of PCA, principal component regression (PCR), attempt to derive concise multidimensional relationships between vectors of inputs and outputs. By directing the information distillation activity onto a set of target outputs, PCR and PLS can form the basis of modelling or virtual metrology methodologies. A useful exposure of PCA, PCR, and PLS in a semiconductor etch setting is given in [32].

A variety of measurements have been used to drive VM models. Chen et al. [33], for example, developed chamber state models of plasma etch using compressed OES data related to etch variables using least squares (LS) on a reactive ion etcher (RIE) with $C_{2} F_{6}$. Data were compressed by manual selection of OES lines, PCA, and partial least squares (PLS) analysis, with model outputs of etch rate uniformity and aspect ratio. In contrast, Tsunami et al. [34] used PIM data in a dual-frequency system to estimate etch rate using a simple linear regression model. However, the model has no drift component and loses accuracy over time. Lee and Spanos [35], on the other hand, use process inputs (pressure, power gas flows, etc.) to build a model for RIE process outputs of etch rate, uniformity, selectivity, and anisotropy. Both PCA and PLS returned prediction errors of 7-10\%. May et al. [36] also used process inputs to estimate etch variables, using response surface modelling. Ragnoli et al. [37] compare VM schemes for etch rate using a variety of techniques, including PCR, PLS, forward selection component analysis (FSCA), and forward selection regression (FSR), based on OES data. FSR and FSCA are found to be more effective for feature selection in OES datasets due to the selection of fewer OES lines to summarize key variations in the process data.

Statistical techniques find wide application in fault detection and classification, typically using a data reduction mechanism such as PCA, and sometimes augmented by a modelling tool such as PLS or neural networks. For example, Shadmehr et al. [38] found OES to be superior for residual gas analysis (using mass spectrometry) and reported success in predicting thin-film contamination levels. Yue et al. [39] also used PCA to detect an etch stop condition in high-density plasma etch. Wise et al. [40] compared PCA with other multivariate methods, including parallel factor analysis and trilinear decomposition, for a metal etch fault detection application. Both local and global models were trained with a variety of sensor data, with local models providing the best performance, using machine state signals and RF information. ICA has, in some cases [31], been shown to outperform PCA in fault detection and classification applications, including semiconductor etch. Using a more traditional time-series approach, Guo et al. [41] use an ARIMA model to filter real-time signals into identically independent and normally distributed components, followed by the application of Hotelling's $T^{2}$ statistic to obtain single scores. The technique was shown to be successful in detecting internal machine variations before wafer product was affected. Forward and reflected powers, in an electron cyclotron resonance (ECR) plasma etch system, were used [42] to aid visualization and fault detection. In particular, forward/reflected power ratios were found to be effective in fault detection and diagnosis. The nonstationarity of etch processes has been directly addressed in [43], where model mean and covariances are adapted during processing, using a version of the exponentially weighted moving average (EWMA) controller. This gave valid operation over six months, including maintenance, cleaning, and new equipment interventions. Summary statistics were also adapted in [44], with a satisfactory Q-statistic over 3000 wafers. Weighted PCA has also been evaluated for use in etch fault detection applications [45].

Endpoint detection is the most popular application of VM, typically using OES measurements, where settings such as integration time can be adapted to optimize signal-to-noise $(\mathrm{S} / \mathrm{N})$ ratio and response times [46]. Early applications [47] used a single OES wavelength, but with decreases in open etch area (down to 1\%), multiple wavelengths are now employed, usually with PCA processing [48]. For example, Rangan et al. [49] used PCA-reduced OES data to form a linear dynamical model, which was shown to be capable of detecting endpoints and transition times in plasma etch. White et al. [50], also using PCA with OES data, used the $T^{2}$ statistic as a measure of model accuracy (i.e., endpoint detection) and the $Q^{2}$ statistic to indicate a need to recompute the PCA basis. Yue et al. [51] propose methods to remove uninformative wavelengths from the PCA model by analyzing variance and thresholding PCA loadings. Fifty wavelengths were retained and gave good endpoint detection. More recent work on sparse PCA [52] holds further possibilities for selecting key wavelengths from OES data. Goodlin's $\mathrm{Ph} . D$. dissertation [53] provides a good overview of statistical methods for OES in endpoint detection and devises a method for weighting OES according to $\mathrm{S} / \mathrm{N}$ ratio.

PIM data can also be useful in endpoint detection, with a number of authors simply using change patterns to indicate the arrival of endpoint [21], [54]. Koh et al. [55] applied PCA to the RF harmonics and demonstrated how the loadings changed as the endpoint is reached. Dewan et al. [25] developed a PIM phase model, the output of which was compared to measured phase for endpoint detection. PIM-based techniques have also been used to optimize cleaning cycles for chemical vapor deposition processes [56]. In Ragnoli et al. [57], nonnegative matrix factorization is employed as a data reduction and variable selection method, where it is applied to OES data for endpoint detection. Results similar to those achieved with PCA were reported.

Imai et al. [58] apply PLS-based VM to detect harmful species using dc bias voltage, while a paper by Khan et al. [59] uses PLS-based virtual metrology to estimate metrology outputs on a run-to-run, rather then a time-series, basis. Actual metrology values are used to update the PLS model recursively, 
and the VM scheme and an associated run-to-run controller are applied to a simulated process, with good results.

Some nonlinear derivatives of linear statistical techniques, such as PCA and PLS, have also been employed in VM for etch processes. In [60], a PCA-based support vector machine (SVM) algorithm, which could loosely be described as a kernel PCA method [61], has been employed for endpoint detection, based on OES measurement. The use of an SVM to facilitate nonlinear PLS in a classification/fault detection setting has also been considered [62], though no applications in plasma etch have yet been reported.

2) Neural Networks: Due to their ability to synthesize nonlinear relationships from process data, ANNs have been widely used in virtual metrology for etch processes, finding application in the prediction of etch variables (etch rate, selectivity, anisotropy, etc), fault classification, and endpoint detection.

The work of Kim [17], [63], [64] is representative in demonstrating how etch rate may be determined from manipulated inputs, such as gas flows, power, pressure, and bias, using ANNs. Typically, a static (nondynamical) map between process inputs and actual (offline) measurements is built up and them subsequently used in a real-time way, with typical prediction errors of 5-7\% reported [17], [63], [65], [66]. A radial basis function network, as opposed to a multilayer perceptron (MLP), was employed by Kim and Park [67] to model etch rate, based on manipulated inputs. They reported a $40 \%$ improvement over some statistical techniques, though Lee and Spanos [35], who compare ANNs to a variety of statistical techniques (LS, PCA, and PLS) could distinguish no improvement, using a wide variety of training signals. Polynomial ANNs were shown by Kim et al. to outperform MLPs for etch rate prediction, using chuck gap, RF power, bias, and $\mathrm{O}_{2}$ fraction as network inputs. ANNs have also been used to produce inverse models for etch rate (i.e., etch rate $\rightarrow$ manipulated inputs), which can be used for real-time control [68]. A paper by Su et al. [69] looks at a variety of ANN architectures against the accuracy and real-time requirements of $\mathrm{R} 2 \mathrm{R}$ process control. They conclude that recurrent ANNs can satisfy requirements and show application to chemical-mechanical polishing (CMP) and etch processes. The issue of input variable selection for ANN-based VM models is dealt with by Lin et al. [70], who use stepwise regression for variable selection from a range of tool variables in an etch process. A similar scheme, using stepwise regression for variable selection with both MLP and radial-basis ANN models, is described in [71] for a chemical vapor deposition process.

ANNs have been employed with OES data, frequently using PCA (or something similar) as a data preprocessing technique. Hong et al. [72] compared the use of PCA and ANNs for feature extraction from OES data, with a further ANN used to model the reduced data. However, 226 "relevant" wavelengths are initially chosen from the 2048, prior to compression. The compression ANN returned seven features while PCA returned five, with comparable results for both, giving prediction errors as low as $0.2 \%$. Kim and Kim [73], however, reported a drastic performance improvement with partial OES models (110 wavelengths) compared to conventional PCA-OES reduction.
Selectivity, the ability to etch one material (e.g., $\mathrm{Si}$ ) at a different rate to another (e.g., photoresist), has also been modelled by ANNs. Himmel and May [65] found ANNs to be superior to quadratic response surfaces, which might be expected since complexity is more limited in the quadratic case. Hong et al. [72] compared ANNs and PCA for data reduction in selectivity prediction, concluding that the ANN reduction was significantly better.

Uniformity, a measure of the spatial variation in etch across the wafer, has also been modelled using ANNs, requiring spatial measurements for model building. Lee and Spanos [35] reported little success in modelling uniformity using manipulated variables (gas flows, power, pressure, and gap) as inputs, while Kim and Kim [73] found that the addition of dc bias (as an input) only served to reduce the accuracy and increase the complexity, of the model. However, Kim et al. [63] reported a prediction error of just $0.4 \%$ using pressure, gas flows, and power as inputs. In the case where OES was used to model nonuniformity alone, PCA was reported [72] to significantly outperform ANN-based data reduction.

ANNs have also been employed to model surface characteristics, such as anisotropy and surface roughness. Hong et al. [72] built an ANN model that modelled anisotropy with an error of less then 2\%, with Kim et al. [63] predicting etch profile angle with an error of less than $4^{\circ}$. However, anisotropy was shown to have a high dependency on RF power. Kim and Kim [73] achieved similar levels of accuracy for etch profile angle, using a reduced set of OES lines, in preference to manipulated inputs, with PCA-OES models also performing poorly. Kim et al. [74] successfully produced an ANN model that predicted the discrepancy in sidewall bottom etch rate compared to center etch rate, using genetic algorithms to optimize the spread values. Surface roughness was modelled, using ANNs, in [75] and [67] using generalized regression and radial basis function networks, respectively. Reference [75] also employed GAs for ANN optimization, while statistical models, for the same application, were found to be significantly inferior in [67].

The classification properties of ANNs have been well documented (e.g., [76]), and fault detection and classification for plasma etch is a significant application area. In one of the few applications of a dynamical ANN for semiconductor manufacturing applications, Hong and May [77] apply a time-series ANN for fault identification. The ANN predicts the manipulated inputs (RF power, pressure, and two gas flows) based on the variations in seven OES lines, six atomic mass signals from a residual gas analyzer, and the sample time index. The sample time index gives a measure of the chamber "age" or usage and should help to account for drift due to chamber residue buildup. The system demonstrated a sensitivity to performance deviations down to $10 \%$. In a similar way, though using a static ANN, Shadmehr et al. [38] used mass spectometry and OES measurements to predict power and gas flows. The ANN was also able to model thin-film contamination levels on the chamber walls. A dynamical radial-basis function ANN was utilized in [78] to predict manipulated inputs, given a window of previous values. The ANN was trained to incorporate normal 


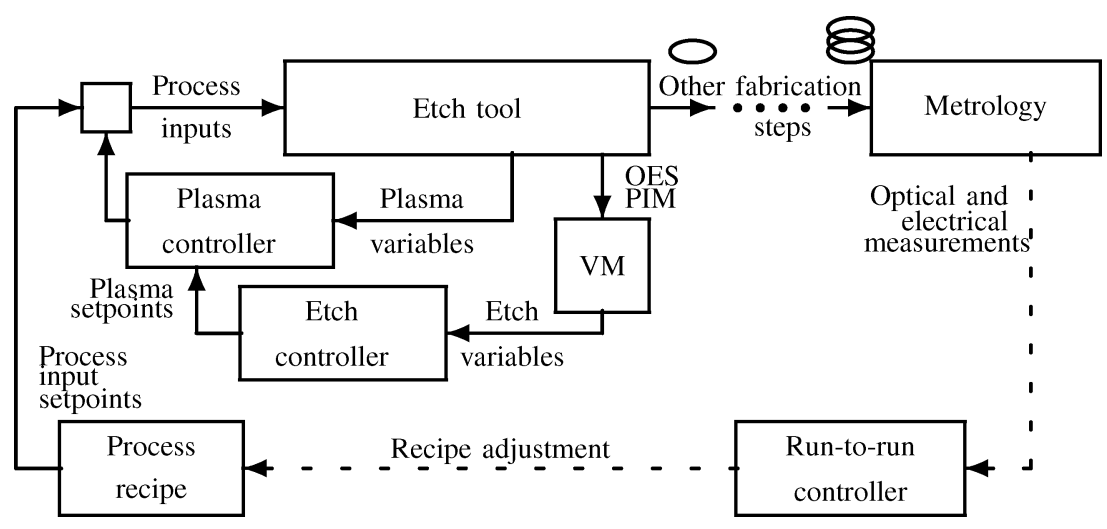

Fig. 2. Etch tool control possibilities.

drift variations and recipe changes and was able to identify abnormal process shifts. A closely related class of paradigm to ANNs, SVMs, which, for certain classes of kernel functions, have a strong similarity with radial basis function neural networks, have also been employed for fault detection, using OES sensor data [79], with $100 \%$ success reported for RF faults in an RIE process.

Endpoint detection has also been addressed using ANNs [80]. The ANN is fed with forward and reflected RF power, matchbox capacitor positions, dc bias, wafer plate power, and previous values of these variables, giving a dynamical context to the classifier input. Target training data was provided by expert (manual) indication of endpoint. Results indicated a strong dependence on a change in the RF signals for successful operation. Other ANN applications include the use of a polynomial ANN to predict the dc bias in an etch chamber [81] and the prediction of overetch time required to control residual oxide thickness [82].

\section{CONTROL}

The control possibilities for a plasma etch tool are outlined in Fig. 2. From a time-domain perspective, the control structures can be divided into the following two general classes:

1) real-time (discrete-time) control, where measurements are immediately used to adjust the manipulated variables that affect the controlled variable(s);

2) run-to-run control, which uses downstream measurements to adjust setpoints for the next wafer or batch of wafers.

For an etch process, two hierarchical real-time control "loops" are possible, as shown in Fig. 2. Plasma variables, such as ion flux and species concentration, may be controlled using an inner loop, where the "recipe" is specified in terms of those quantities and/or an outer loop may be employed to control etch variables (such as rate and depth) directly. Both loops however, depend on measurements of the appropriate controlled variables, which can be supplied by sensors or using virtual metrology. The important subject of measurement is dealt with in detail in Section III. For run-ro-run (R2R) control, the immediate provision of measurements is not so crucial, since $R 2 R$ addresses longer term disturbances, such as residue buildup, etc., and is based on long-term statistical averages.
One must also be mindful of the possible negative impact of the "inner" real-time control loop on the "outer" run-to-run control loop, since the statistical process control (SPC) principles of run-to-run control are based on building up a consistent picture of the statistical process variations. The real-time control normally operates somewhat independently of the run-to-run control, and it is often recommended not to use both approaches simultaneously since, if there is an assignable cause that can be eliminated using SPC, real-time control can mask its effect by making constant adjustments to the manipulated variable. Such masking can, in turn, lead to instability in the process. For more information on this interaction, see [83] and [84].

\section{A. Real-Time Control}

Some of the early work on real-time control, sometimes termed engineering process control, for etch processes looks at control variable selection and the design of a multivariable steady-state compensator [85], [86]. Relative gain array and singular value decomposition methods were used to understand the relationship between process variables (i.e., dc bias, species concentration, etc.), etch variables (yield, uniformity, anisotrophy), and manipulated variables (pressure, power, gas flow rates). This permitted the multivariable system to be approximately decoupled using a static $2 \times 2$ compensator, with single-input single-output (SISO) controllers used for dynamic control. The issue of variable selection/matching has also been considered by Patterson et al. in [87] and [88].

1) Control of Plasma Variables: In this section, the control of plasma variables (such as ion flux and the concentration of various plasma species) is considered, using causal inputs such as RF power, pressure (via valve position), and gas flow rates. In [16], [89], and [90], for example, fluorine concentration $[F]$ and $V_{\text {bias }}$ are controlled in real time using power and pressure as manipulated variables. Measurement of $[F]$ was via OES with actinometry, while an inductive tap was used to measure $V_{\text {bias }}$. A $2 \times 2$ transfer function matrix for the system was identified and a control algorithm based on linear quadratic Gaussian with loop transfer recovery (LQG/LTR) methods employed. A Hammerstein model component was also added in [91] and [92] to allow static actuator nonlinearities to be accounted for; [92] also addresses the issue of spatial uniformity on the wafer. A control system that regulates the ion energy (measured using a Langmuir probe) in a capacitively coupled RIE is reported in [93]. 
$V_{\text {bias }}$ was adjusted using a resistor in parallel with the blocking capacitor. It was deliberately chosen not to use RF power and gas concentrations as control variables, since these affect other aspects of the etch. Two applications, selectivity enhancement and ion-enhanced plasma cleaning, were shown to benefit from the control, though a reduction in plasma stability was noted. A slightly different approach is presented in [94], where ion current and ion energy are controlled via RF power and bias power using two SISO PI controllers. Ion current is estimated from antenna impedance, while ion energy is inferred from root mean square RF voltage. A decoupling compensator, controlling ion flux and species concentration in a variety of $\mathrm{Ar} / \mathrm{O}_{2} / \mathrm{C}_{4} \mathrm{~F}_{8}$ plasmas, is presented in [95]. In particular, the multivariable interaction between the manipulated variables (RF power, gas flows) is addressed. More recently, Lin et al. [96] describe a scheme where RF power is manipulated to control electron density and ion energy, where a transmission line microstrip microwave inferometer is used to measure electron density. This scheme is shown to achieve good consistency in etch characteristics and can counteract the "first wafer" effect. A fuzzy logic controller was shown to outperform a traditional PI controller.

Hanish et al. [97] demonstrate one of the few applications of an asymptotic observer, an extended Kalman filter (EKF), which is used to estimate $[C l]$ from OES with actinometry. Two SISO PID controllers are used to control $[C l]$ and pressure, using the rate constant of dissociation and "gas loss" (essentially outlet flow plus gas loss to chamber walls) as manipulated variables. A PID controller is also employed by Klimecky et al. [98] to control plasma density, using RF power as a manipulated variable. One of the significant features is the claim that the controller can compensate for transient chamber wall conditions and therefore provide better consistency in the face of residue buildup.

2) Control of Etch Variables: In this section, the control of etch variables, either directly or indirectly, is considered. An interesting paper by Vincent et al. [99] employs an EKF to measure etch rate, using dual-frequency reflectometry as a correcting output. The focus is on the use of power, as a manipulated variable, to control etch rate, using a PI controller, though other control structures could be considered. The authors report an $83 \%$ improvement in etch depth results compared to a purely timed etch. A model-based feedback controller is reported in [68] and [100], controlling etch rate, which is measured using laser inferometry and a profilometer. Manipulated variables are pressure, RF power, and gas flow, and a linear LQG/LTR controller is compared to a nonlinear adaptive controller based on a neural network model. The nonlinear adaptive controller is shown to be superior under parameter variation and disturbance conditions. Armaou et al. [101] describe a PI-based control scheme that manipulates gas flows to control three spatially distributed measurements of etch rate. Results are presented for a simulation and demonstrate a potential reduction in etch-rate nonuniformity from $30.2 \%$ to $3.8 \%$.

In [102], the control system is extended to include the control of $V_{\text {bias }}$. Etch rate of GaAs, measured using spectroscopic ellipsometry, is also controlled in [103]. A model-based LQG controller is used to manipulate pressure, where the model is determined by fitting experimental data to a first-principles structure. This paper also presents a robust adaptive controller for $\mathrm{aSi}_{3} \mathrm{~N}_{4}$ etch application based on an empirical model. The use of phase difference between upper and lower RF signals as a manipulated input acting on etch characteristics has been demonstrated by Sung et al. [104] and could provide the basis for real-time control.

\section{B. Run-to-Run Control}

In contrast to real-time control, R2R control works with delayed measurements and is driven by the availability of wafer measurements. It therefore falls into the class of discrete-event rather then discrete-time (as is the case of real-time control) systems. An excellent overview of the application of R2R control in the semiconductor industry is given in the book by Moyne et al. [105]. R2R control has its origins in SPC, which provides tools for the detection of process faults using statistical techniques. $\mathrm{R} 2 \mathrm{R}$ extends these ideas to provide active control via feedback.

1) Algorithms: Since there are a small number of $\mathrm{R} 2 \mathrm{R}$ algorithms, mostly based around the EWMA controller [84], we give the SISO double EWMA controller (which can cater for both slow drifts and fast shocks) [106] as

$$
\begin{aligned}
Y_{t}= & a+b X_{t-1}+d \cdot t+\varepsilon_{t} \\
\hat{a}_{t}= & \lambda_{1}\left(Y_{t}-b X_{t-1}\right)+\left(1-\lambda_{1}\right) \hat{a}_{t-1} \\
\hat{d}_{t}= & \lambda_{2}\left(Y_{t}-b X_{t-1}-\hat{a}_{t-1}\right)+\left(1-\lambda_{2}\right) \hat{d}_{t-1} \\
& 0<\lambda_{1}, \lambda_{2} \leq 1 \\
X_{t}= & \frac{\bar{Y}-\hat{a}_{t}-\hat{d}_{t}}{\hat{b}}
\end{aligned}
$$

where (3) defines the process model, (4) and (5) are estimators for the model parameter and drift, respectively, with (6) calculating the manipulated value. $Y_{t}$ is the controlled variable (e.g., etch depth), $\bar{Y}$ is the setpoint for $Y_{t}, X_{t}$ is the manipulated variable, $a$ and $b$ (which is determined a priori) are model parameters, and $\varepsilon_{t}$ is a disturbance. The " " notation is used to denote an estimate, and $\lambda_{1}$ and $\lambda_{2}$ are tuning parameters used to specify the sensitivity to variations in $a_{t}$ and $d_{t}$, respectively. The choice of $\lambda_{1}$ and $\lambda_{2}$ is discussed in [84]. Note that the double EWMA controller assumes a linear drift in the process, of the form $d \cdot t$, and implements a form of deadbeat control [107], which attempts to regulate the process output in minimum time (samples).

Since EWMA controllers are based on statistical measures of process behavior, their use with mixed products needs to be considered carefully. One solution to the mixed product problem include the employment of a disturbance model for $\hat{d}_{t}(i)$ for each product type $i$, with the appropriate $\hat{d}_{t}(i)$ used in the deadbeat control calculation in $X_{t}$ [108], [109]. EWMA controllers can also be used across different tools, via the use of multiple process models [108], by employing different $a$ and $b$ parameters for each tool. Zheng et al. [108] also consider the stability and performance implications of using multiple process and disturbance models.

2) Model Identification: While (4) provides an estimate for a, Wang et al. [110] prefer a Bayesian statistic for the detection and classification of disturbances in order to obtain better parameter estimation and illustrate its use on a film deposition process. An additional common method for parameter estimation of linear models is least squares (LS), frequently used also 
in R2R control [111]-[115] (simulated CMP process). In this case, (3) is replaced by a recursive LS estimator for the online estimation of the intercept $a$, while a weighted LS algorithm [116] allows the assignment of preference to current measurements versus past measurements. Palmer et al. [117] employ a Kalman filter to estimate both the gain and the intercept and apply it to a photoresist deposition process. The "age-based" double EWMA controller presented in [118] introduces a term into the model to represent a drift due to the tools' wearing, which is proportional to the number of runs since the last maintenance operation.

3) Weight Determination: The performances of EWMA-type controllers are strongly affected by the choice of the weights $\lambda_{1}$ and $\lambda_{2}$ (also called "forgetting factors") [84], [119]. Discussion and methods concerning the optimal choice of weights can be found in [120] (CMP application), [121], and [114], while in [122], the choice of the weights is studied with respect to the performance tradeoff between the short-term and long-term responses of the system. In [123], weight optimization trades off controlled variable regulation against manipulated variable effort, using a heuristic algorithm. A similar strategy has been adopted in [124], where the minimization focuses on the regulated outputs, using dynamic programming theory. In [125], a neural network is used to map the relation between the disturbance state (drift and noise) and the optimal weight of an EWMA estimator.

4) Multivariable Models: Modifications to the basic EWMA controller, with etch applications, include extensions to allow multivariable models [84], nonsquare models [105], and nonlinear models [114]. With regard to nonsquare systems, there are two possibilities:

a) more output (controlled) variables than available manipulated variables (underactuated);

b) more manipulated variables than outputs (overactuated). In the case of a), a "best compromise" solution can be achieved using least squares type solutions (see [105]). One possibility is also to attach a greater importance to the regulation of one or more controlled variables, as exemplified for the chemical-mechanical polishing (CMP) case in [126]. In the overactuated case, some additional freedom is available to the control designer, over and above regulating the controlled variables. One way to exploit this freedom, seen in a variety of control applications, is to minimize the energy or variance of the control action. This is vital in some applications, such as aerospace, where limited energy to power actuators is available, but could also encourage less actuator wear and material usage in semiconductor etch. Examples of how overactuated systems are dealt with include:

- weight assignment to minimize the variance of specific manipulated variables (for a CMP process) [120];

- the use of Lagrange multipliers for new recipe calculation (silicon epitaxy and CMP applications) [121];

- the use of a nonsquare matrix pseudoinverse (which can be related to least squares minimization of the manipulated variables) [119];

- the use of PLS to determine a diagonalizing (nonsquare) precompensator, followed by application of multiple single-loop double EWMA controllers [127] (also for a CMP application);

- ridge regression [128], where a bound is put on manipulated signal values.

The paper by Khan et al. [59] uses a multivariable EWMA controller based on a PLS model in both wafer-to-wafer and lot-to-lot control, where an advantage in wafer-to-wafer control is highlighted.

Bounding the manipulated input signal, in addition to potentially reducing actuator wear and energy use, also has the advantage of respecting actuator constraints (both amplitude and rate), reducing the incidence of nonlinear behavior. In addition to the input bounding method of Rajagopal and del Castillo [128], Boning et al. proposed the recursive bound pinning technique [120] (demonstrated on a CMP application).

5) Nonlinear Models: Nonlinear models give the possibility of obtaining a better description of the process, which can subsequently improve the R2R control performance. Using static (nondynamical) polynomial elements, it is possible to introduce nonlinearity in the model while retaining the ability to employ recursive LS estimation techniques, as in [115], [114], and [111], since the model is still linear in the parameters. A second-order polynomial is employed in [106] and [112], where the estimation technique is based on a steady-state design of experiments (DOE). A different approach has been taken in [129], where a bounding ellipsoid algorithm is employed to estimate parameters of a second-order polynomial model. At each iteration, the algorithm returns an outer bounding ellipsoid of the likely process parameter set. Ramaswamy et al. [130] designed a variable gain controller for the compensation of nonlinearities, which solves a min-max problem to find the input adjustment that minimizes the worst case predicted error using a double EWMA controller.

Neural networks are also widely used due to their ability to model nonlinear systems; however, a large number of samples are generally required for training. This is not a substantial issue in high-volume manufacturing, such as semiconductor etch, since most production plants are equipped with extensive databases for the recording of large numbers of process variables. Such data have been used in [82], [123], and [131]-[133] to train a neural network for the mapping of etch process input/ output relationships. A neural network model, predicting etch rate, is combined with a real-time optimizer in [131] to provide process setpoints to alleviate long-term process drift and sensitivity to PM interventions. The ANN model is trained with extensive historical process data prior to use. By comparison, in [91] and [134], the data for the training of the neural network are collected by performing a specific experiment using DOE techniques.

6) Other R2R Control Structures: Though EWMA controllers are among the most popular in semiconductor process control, a number of other structures have been investigated. Controllers based on disturbance feedforward adjust the recipe in accordance with the outcome of upstream processes, with etch applications reported in [135] and [136]. Another type of "lookup" controller interrogates historical data to look for a similar previous situation or interpolates to find the 
closest match [137]. Both of these controller types provide more conservative options than R2R algorithms, which allow batch-to-batch adjustment and are common in many production environments.

Using intelligent systems methods, fuzzy logic and database learning have been incorporated into a generic R2R etch controller in [138], while genetic algorithms for recipe generation and low variance control have been used in [139] and [140]. A multiresolution reinforcement learning approach to $\mathrm{R} 2 \mathrm{R}$ control was also examined in [141].

Model predictive control, which has found widespread application in real-time process control, has also been extended to R2R control in [142] and [143], while Hamby et al. [144] adopted a probabilistic approach to R2R control.

A number of other issues relating to R2R control, such as stability, robustness, and measurement delay, are also important and have received some attention in the published literature but are omitted here for brevity. The interested reader is directed to [10] for an overview.

\section{CONCLUSION}

Since plasma etch in semiconductor processing is such a high-value process, it has attracted significant attention in attempts to improve the process quality and consistency. However, in most production environments, such processes are effectively run in "open-loop" mode, with reliance on consistency in recipe and etch chamber state. In some instances, some schedule-based correction to the recipe is applied to account for process drift due to residue deposition on the chamber walls. While a number of run-to-run applications are reported, particularly in experimental setups, there is a paucity of documented application of real-time control of process variables. The principal reason for this is that direct measurement of key etch variables is difficult (Langmuir probes are invasive, while reflectometry is notoriously difficult in a production environment), with virtual metrology providing a promising alternative, using indirect measurements (such as OES and PIM) as a basis. However, most current VM strategies use only a forward model, unlike the predictor-corrector structure, which is characteristic of linear state estimators. This could provide a possibility for improvement of VM algorithms. With the availability of reliable measurements, there is an enormous range of control algorithms that could be brought to bear [145], [146]. Predictive control, for example, has many desirable characteristics and has been widely applied in industrial process control settings [147].

One real-time control possibility is to attempt to directly control plasma variables, such as ion flux and species density, and to base a "recipe" on these variables, which may help to achieve consistency in etch, in an environment experiencing drift (residue buildup) and step changes (PM steps). Run-to-run control for etch still has a number of important challenges, some of which arise from the delay and others related to the uncertainty associated with the effect of other processing steps between actuation and measurement. The delay may result in significant product wastage before corrective action is taken and provides a stability challenge for the control engineer, while the presence of intermediate process steps between actuation and measurement creates a difficulty in relating effect to cause. However, run-to-run control algorithms appear to have mainly converged to variants of the EWMA type.

The selection of key variables is an important issue. Indirect measurements, such as PIM and OES signals, are high in dimension, and appropriate dimension reduction is vital if etch variables (such as etch rate) are to be reconstructed using virtual metrology. Likewise, selection of key manipulated variables is important since the plasma etch process is a highly interactive multivariable nonlinear system and care must be taken that only the desired controlled variables will be affected by feedback control action.

\section{ACKNOWLEDGMENT}

The authors are grateful for the contribution to this work by N. MacGearailt of Intel (Ireland) Ltd.

\section{REFERENCES}

[1] P. Otellini, Intel Corp., Intel developer forum Sep. 18, 2007 [Online]. Available: www.intel.com, Tech. Rep.

[2] S. Limanond, J. Si, and K. Tsakalis, "Monitoring and control of semiconductor manufacturing processes," IEEE Control Syst. Mag., vol. 18, no. 6 , pp. $46-58,1998$.

[3] A. Emami-Naeini, J. Ebert, R. Kosut, D. de Roover, and S. Ghosal, "Model-based control for semiconductor and advanced materials processing: An overview," in Proc. Amer. Contr. Conf., Boston, MA, Jun. 2004, vol. 5, pp. 3902-3909.

[4] A.-J. Su, J.-C. Jeng, H.-P. Huang, C.-C. Yu, S.-Y. Hung, and C.-K. Chao, "Control relevant issues in semiconductor manufacturing: Overview with some new results," Contr. Eng. Practice, vol. 15, no. 10, pp. 1268-1279, 2007.

[5] G. S. May and C. J. Spanos, Fundamentals of Semiconductor Manufacturing and Process Control. New York: Wiley-IEEE Press, 2006.

[6] S. J. Qin, G. Cherry, R. Good, J. Wang, and C. A. Harrison, "Semiconductor manufacturing process control and monitoring: A fab-wide framework," J. Process Contr., vol. 16, no. 3, pp. 179-191, 2006.

[7] T. Edgar, S. Butler, W. Campbell, C. Pfeiffer, C. Bode, S. Hwang, K. Balakrishnan, and J. Hahn, "Automatic control in microelectronics manufacturing: Practices, challenges, and possibilities," Automatica, vol. 36, no. 11, pp. 1567-1603, 2000.

[8] J. Moyne and N. Patel, "Special section on advanced process control," IEEE Trans. Semicond. Manuf., vol. 20, no. 4, pp. 343-344, Nov. 2007.

[9] S. Lynn, Virtual metrology for plasma etching: A literature review Dept. of Electrical Engineering, NUI Maynooth, Ireland, Res. Rep. $\mathrm{EE} / \mathrm{JVR} / 4 / 2007,2007$.

[10] G. Bacelli, Control in semiconductor manufacturing: A literature review Dept. of Electrical Engineering, NUI Maynooth, Ireland, Res. Rep. EE/JVR/3/2007, 2007, Tech. Rep..

[11] M. Beibei, Literature review: Endpoint detection in plasma etching Dept. of Elect. Eng., NUI Maynooth, Ireland, Res. Rep. EE/SMcL/1/ 2007, 2007.

[12] J. P. Verboncoeur, "Particle simulation of plasmas: Review and advances," Plasma Phys. Contr. Fusion, vol. 47, no. 5A, pp. A231-A260, 2005.

[13] M. J. Kushner, "A kinetic study of the plasma-etching process-I: A model for the etching of $\mathrm{Si}$ and $\mathrm{SiO}_{2}$ in $\mathrm{C}_{n} \mathrm{~F}_{m} / \mathrm{H}_{2}$ and $\mathrm{C}_{n} \mathrm{~F}_{m} / \mathrm{O}_{2}$ plasmas," J. Appl. Phys., vol. 4, pp. 2923-2938, 1982.

[14] E. Gogolides, M. Stathakopoulos, and A. Boudouvis, "Modelling of radio frequency plasmas in tetrafluoromethane $\left(\mathrm{CF}_{4}\right)$ : The gas phase physics and the role of negative ion detachment," J. Phys. D, Appl. Phys., vol. 27, no. 9, pp. 1878-1886, 1994.

[15] M. A. Sobolewski, "Real-time, noninvasive monitoring of ion energy and ion current at a wafer surface during plasma etching," $J$. Vac. Sci. Technol., vol. 24, no. 5, pp. 1892-1905, 2006.

[16] B. Rashap, P. Khargonekar, J. Grizzle, M. Elta, J. Freudenberg, and F. Terry, "Real-time control of reactive ion etching: Identification and disturbance rejection," in Proc. 32nd IEEE Conf. Decision Contr., 1993, pp. 3379-3385.

[17] B. Kim and B. T. Lee, "Prediction of silicon oxynitride plasma etching using a generalized regression neural network," J. Appl. Phys., vol. 98, no. 3 , p. $034912,2005$. 
[18] S. H. Lee, F. Iza, and J. K. Lee, "Particle-in-cell monte carlo and fluid simulations of argon-oxygen plasma: Comparisons with experiments and validations," Phys. Plasmas, vol. 13, no. 5, p. 057102, 2006.

[19] L. Ljung, System Identification: Theory for the User. Englewood Cliffs, NJ: Prentice-Hall, 1999.

[20] P. Iordanov and J. Ringwood, "Control-oriented modelling of an experimental $\mathrm{Ar}_{-} \mathrm{O}_{2}$ plasma process," in Proc. IEEE-ICIT Intl. Conf. Ind. Tech., Valparaiso, Chile, Mar. 2010.

[21] M. Kanoh, M. Yamage, and H. Takada, "End-point detection of reactive ion etching by plasma impedance monitoring," Jpn. J. Appl. Phys., vol. 40, no. 3A, pp. 1457-1462, 2001

[22] M. A. Sobolewski, "Noninvasive monitoring of ion energy drift in an inductively coupled plasma reactor," J. Appl. Phys., vol. 97, no. 3, pp. 033 301-1-033 301-12, 2005.

[23] Y. Yang, M. Wang, and M. J. Kushner, "Progress, opportunities and challenges in modeling of plasma etching," in Proc. Int. Interconnect Technol. Conf. (IITC), Sapporo, Japan, Jun. 2008, pp. 90-92.

[24] Straatum, Scientific Systems Ltd., Knowledge based process control 2002 [Online]. Available: http://www.straatum.com/

[25] M. N. A. Dewan, P. J. McNally, T. Perova, and P. A. F. Herbert, "Use of plasma impedance monitoring for determination of sf6 reactive ion etching end point of the sio2/si system," Microelectron. Eng., vol. 65, no. $1-2$, pp. $25-46,2003$.

[26] R. Kalman and R. Bucy, "New results in linear filtering and prediction theory," ASME J. Basic Eng. D, vol. 83, pp. 95-108, 1961.

[27] S. Lynn, J. Ringwood, E. Ragnoli, S. McLoone, and N. MacGearailt, "Virtual metrology for plasma etch using tool variables," in Proc. IEEE/ SEMI Adv. Semicond. Manuf. Conf. (ASMC), Berlin, May 2009, pp. $143-148$.

[28] I. Jolliffe, Principal Component Analysis. New York: SpringerVerlag, 1986.

[29] P. Comon, "Independent component analysis-A new concept?," Signal Process., vol. 36, pp. 287-314, 1994.

[30] J.-F. Cardoso, "Blind signal separation: Statistical principles," Proc. IEEE, vol. 86, pp. 2009-2025, Oct. 1998.

[31] J.-M. Lee, S. J. Qin, and I.-B. Lee, "Fault detection and diagnosis based on modified independent component analysis," AIChE J., vol. 52, no. 10, pp. 3501-3514, 2006.

[32] D. White, D. Boning, S. Watts Butler, and G. Barna, "Spatial characterization of wafer state using principal component analysis of optical emission spectra in plasma etch," IEEE Trans. Semicond. Manuf., vol. 10, no. 1, pp. 52-61, Feb. 1997.

[33] R. Chen, H. Huang, C. J. Spanos, and M. Gatto, "Plasma etch modeling using optical emission spectroscopy," J. Vac. Sci. Technol. A, Vac. Surf. Films, vol. 14, no. 3, pp. 1901-1906, 1996.

[34] D. Tsunami, J. McNames, B. Whitefield, P. Rudolph, and J. Zola, "Oxide etch rate estimation using plasma impedance monitoring," in Proc. SPIE, 2005, vol. 5755, pp. 59-68.

[35] S. Lee and C. Spanos, "Prediction of wafer state after plasma processing using real-time tool data," IEEE Trans. Semicond. Manuf., vol. 8, no. 3, pp. 252-261, Aug. 1995

[36] G. May, J. Huang, and C. Spanos, "Statistical experimental design in plasma etch modeling," IEEE Trans. Semicond. Manuf., vol. 4, no. 4, pp. 83-98, Nov. 1991

[37] E. Ragnoli, S. McLoone, J. Ringwood, and N. Macgerailt, "Identifying key process characteristics and predicting etch rate from high-dimensional datasets," in Proc. IEEE/SEMI Adv. Semicond. Manuf. Conf. (ASMC), Berlin, 2009, pp. 106-111.

[38] R. Shadmehr, D. Angell, P. B. Chou, G. S. Oehrlein, and R. S. Jaffe, "Principal component analysis of optical emission spectroscopy and mass spectrometry: Application to reactive ion etch process parameter estimation using neural networks," J. Electrochem. Soc., vol. 139, no. 3, pp. 907-914, 1992

[39] H. Yue, S. Qin, R. Markle, C. Nauert, and M. Gatto, "Fault detection of plasma etchers using optical emission spectra," IEEE Trans. Semicond. Manufact., vol. 13, no. 3, pp. 374-385, Aug. 2000.

[40] B. M. Wise, N. B. Gallagher, D. D. Butler, S. W. White, and G. G. Barna, "A comparison of principal component analysis, multiway principal component analysis, trilinear decomposition and parallel factor analysis for fault detection in a semiconductor etch process," J. Chemometrics, vol. 13, no. 3-4, pp. 379-396, 1999.

[41] H.-F. Guo, C. Spanos, and A. Miller, "Real time statistical process control for plasma etching," in Proc. IEEE/SEMI Semicond. Manuf. Sci. Symp., 1991, pp. 113-118.

[42] V. J. Law and N. Macgearailt, "Visualization of a dual-frequency plasma etch process," Meas. Sci. Technol., vol. 18, no. 3, pp. 645-649, 2007.

[43] N. Gallagher and B. Wise, Eigenvector Research Inc., Development and benchmarking of multivariate statistical process control tools for a semiconductor etch process: Improving robustness through model updating Tech. Rep., 1997.
[44] K. A. Chamness and T. Edgar, "Diagnostics of plasma etch: PCA with adaptive centering and scaling," in Proc. AEC/APC XV Symp., 2003.

[45] H. Yue and M. Tomoyasu, "Weighted principal component analysis and its applications to improve fdc performance," in Proc. IEEE Conf. Decision Contr., 2004, pp. 4262-4267.

[46] S. Thomas, III, H. H. Chen, C. K. Hanish, J. W. Grizzle, and S. W. Pang, "Minimized response time of optical emission and mass spectrometric signals for optimized endpoint detection," J. Vac. Sci. Technol. $B$, vol. 14, no. 4, pp. 2531-2536, 1996.

[47] S. Dolins, A. Srivastava, and B. Flinchbaugh, "Monitoring and diag nosis of plasma etch processes," IEEE Trans. Semicond. Manuf., vol. 1, no. 1, pp. 23-27, Feb. 1988

[48] K. Han, E. S. Yoon, J. Lee, H. Chae, K. H. Han, and K. J. Park, "Realtime end-point detection using modified principal component analysis for small open area sio2 plasma etching," Ind. Eng. Chem. Res., vol. 47, no. 11 , pp. 3907-3911, 2008

[49] S. Rangan, C. Spanos, and K. Poolla, "Modeling and filtering of optical emission spectroscopy data for plasma etching systems," in Proc. IEEE Int. Symp. Semicond. Manuf., 1997, pp. B41-B44.

[50] D. White, B. Goodlin, A. Gower, D. Boning, H. Chen, H. Sawin, and T. Dalton, "Low open-area endpoint detection using a PCA-based $\mathrm{T}^{2}$ statistic and Q statistic on optical emission spectroscopy measurements," IEEE Trans. Semicond. Manuf., vol. 13, no. 2, pp. 193-207, 2000.

[51] H. H. Yue, S. J. Qin, J. Wiseman, and A. Toprac, "Plasma etching endpoint detection using multiple wavelengths for small open-area wafers," J. Vac. Sci. Technol. A, vol. 19, no. 1, pp. 66-75, 2001.

[52] A. d'Aspremont, L. E. Ghaoui, M. Jordan, and G. R. G. Lanckriet, "A direct formulation for sparse PCA using semidefinite programming," SIAM Rev., vol. 49, no. 3, pp. 434-448, 2007.

[53] B. E. Goodlin, "Multivariate endpoint detection of plasma etching processes," Ph.D. dissertation, Univ. of Texas, Austin, Apr. 2002.

[54] V. Patel, B. Singh, and J. H. Thomas, "Reactive ion etching end-point determination by plasma impedance monitoring," Appl. Phys. Lett., vol. 61, no. 16, pp. 1912-1914, 1992.

[55] A. Koh, N. Thornhill, and V. Law, "Principal component analysis of plasma harmonics in end-point detection of photoresist stripping," Electron. Lett., vol. 35, no. 16, pp. 1383-1385, 1999.

[56] M. Bonner and R. Clark, "RF-based sensor technology improves cleaning efficiency on pecvd tools," Semicond. Fabtech, vol. 19, pp. $1-4,2003$.

[57] E. Ragnoli, S. McLoone, J. Ringwood, and N. Macgerailt, "Matrix factorisation techniques for endpoint detection in plasma etching," in Proc. IEEE/SEMI Advanced Semicond. Manuf. Conf. (ASMC), Cambridge, MA, 2008, pp. 156-161.

[58] S. Imai, "Virtual metrology for plasma particle in plasma etching equipment," in Proc. Int. Symp. Semicond. Manuf. (ISSM), Tokyo, Japan, 2007 [Online]. Available: http://ieeexplore.ieee.org/stamp/stamp. jsp?arnumber $=04446835$, Paper PC-O-186

[59] A. A. Khan, J. Moyne, and D. Tilbury, "Virtual metrology and feedback control for semiconductor manufacturing processes using recursive partial least squares," J. Process Contr., vol. 18, no. 10, pp. 961-974, 2008

[60] K. Han, S. Kim, K. J. Park, E. S. Yoon, and H. Chae, "Principal component analysis based support vector machine for the end point detection of the metal etch process," in Proc. IFAC World Congr., Seoul, Korea.

[61] J.-M. Lee, C. Yoo, and I.-B. Lee, "Fault detection of batch processes using multiway kernel principal component analysis," Comput. Chem. Eng., vol. 28, no. 9, pp. 1837-1847, 2004.

[62] R. Rosipal, L. Trejo, and B. Matthews, "Kernel pls-svc for linear and nonlinear classification," in Proc. 20th Int. Conf. Machine Learn. (ICML), Washington, DC, 2003, pp. 218-223.

[63] B. Kim, K. Kwon, S. Kwon, J. Park, S. Yoo, K. Park, I. You, and B. Kim, "Modeling etch rate and uniformity of oxide via etching in a $c h f_{3} / c f_{4}$ plasma using neural networks," Thin Solid Films, vol. 426 , no. $1-2$, pp. $8-15,2003$

[64] B. Kim and B.-T. Lee, "Effect of plasma and control parameters on sic etching in a $c_{2} f_{6}$ plasma," Plasma Chem. Plasma Process., vol. 23, no. 3, pp. 489-499, 2003.

[65] C. Himmel and G. May, "Advantages of plasma etch modeling using neural networks over statistical techniques," IEEE Trans. Semicond. Manuf., vol. 6, no. 2, pp. 103-111, May 1993.

[66] B. Kim, W. Choi, and H. Kim, "Using neural networks with a linear output neuron to model plasma etch processes," in Proc. IEEE Int. Symp. Ind. Electron., 2001, vol. 1, pp. 441-445.

[67] B. Kim and K. Park, "Modeling plasma etching process using a radial basis function network," Microelectron. Eng., vol. 77, no. 2, pp. $150-157,2005$

[68] D. Stokes and G. May, "Real-time control of reactive ion etching using neural networks," IEEE Trans. Semicond. Manuf., vol. 13, no. 4, pp. 469-480, Dec. 2000. 
[69] Y.-C. Su, T.-H. Lin, F.-T. Cheng, and W.-M. Wu, "Accuracy and realtime considerations for implementing various virtual metrology algorithms," IEEE Trans. Semicond. Manuf., vol. 21, no. 3, pp. 426-434, Aug. 2008.

[70] T.-H. Lin, F.-T. Cheng, W.-M. Wu, C.-A. Kao, A.-J. Ye, and F.-C. Chang, "Nn-based key-variable selection method for enhancing virtual metrology accuracy," IEEE Trans. Semicond. Manuf., vol. 22, no. 1, pp. 204-211, Feb. 2009.

[71] A. Ferreira, A. Roussy, and L. Conde, "Virtual metrology models for predicting physical measurement in semiconductor manufacturing," in Proc. IEEE/SEMI Adv. Semicond. Manuf. Conf., Berlin, 2009, pp. $149-154$.

[72] S. Hong, G. May, and D.-C. Park, "Neural network modeling of reactive ion etching using optical emission spectroscopy data," IEEE Trans. Semicond. Manuf., vol. 16, no. 4, pp. 598-608, Nov. 2003.

[73] B. Kim and S. Kim, "Partial diagnostic data to plasma etch modeling using neural network," Microelectron. Eng., vol. 75, no. 4, pp. 397-404, 2004.

[74] B. Kim, D. Han, S. Moon, and K. K. Lee, "Modeling of sidewall bottom etching using a neural network," J. Korean Phys. Soc., vol. 46, no. 6, pp. 1365-1370, 2005.

[75] B. Kim and K. Kim, "Prediction of profile surface roughness in chf3/cf4 plasma using neural network," Appl. Surface Sci., vol. 222, no. $1-4$, pp. $17-22,2004$.

[76] S. Huang and K. K. Tan, "Fault detection and diagnosis based on modeling and estimation methods," IEEE Trans. Neural Netw., vol. 20, no. 3, pp. 872-881, May 2009.

[77] S. Hong and G. May, "Neural-network-based sensor fusion of optical emission and mass spectroscopy data for real-time fault detection in reactive ion etching," IEEE Trans. Ind. Electron., vol. 52, no. 4, pp. 1063-1072, Jul./Aug. 2005

[78] Y.-J. Chang, "Fault detection for plasma etching processes using RBF neural networks.," in Proc. ISNN, 2005, pp. 538-543.

[79] T. Sarmiento, S. Hong, and G. May, "Fault detection in reactive ion etching systems using one-class support vector machines," in IEEE/ SEMI Adv. Semicond. Manuf. Conf. Workshop, 2005, pp. 139-142.

[80] H. L. Maynard, E. A. Rietman, J. T. C. Lee, and D. E. Ibbotson, "Plasma etching endpointing by monitoring radio-frequency power systems with an artificial neural network," J. Electrochem. Soc., vol. 143, no. 6, pp. 2029-2035, 1996.

[81] B. Kim, D. W. Kim, and G. T. Park, "Prediction of plasma etching using a polynomial neural network," IEEE Trans. Plasma Sci., vol. 31, no. 6, pp. 1330-1336, Dec. 2003.

[82] E. Rietman and S. Patel, "A production demonstration of wafer-towafer plasma gate etch control by adaptive real-time computation of the over-etch time from in situ process signals," IEEE Trans. Semicond. Manuf., vol. 8, no. 3, pp. 304-308, Aug. 1995.

[83] G. Box, A. Luceno, and M. Paniagua-Quinones, Statistical Control by Monitoring and Adjustment, 2nd ed. New York: Wiley, 2009.

[84] E. del Castillo, Statistical Process Adjustment for Quality Control. New York: Wiley, 2002.

[85] S. W. Butler, K. J. McLaughlin, T. F. Edgar, and I. Trachtenberg, "Development of techniques for real-time monitoring and control in plasma etching," J. Electrochem. Soc., vol. 138, no. 9, pp. 2727-2735, 1991.

[86] K. McLaughlin, T. Edgar, and I. Trachtenberg, "Real-time monitoring and control in plasma etching," IEEE Contr. Syst. Mag., vol. 11, no. 3, pp. 3-10, 1991.

[87] O. Patterson, X. Dong, P. Khargonekar, and V. Nair, "Methodology for feedback variable selection for control of semiconductor manufacturing processes-Part 1: Analytical and simulation results," IEEE Trans. Semicond. Manuf., vol. 16, no. 4, pp. 575-587, Nov. 2003.

[88] O. Patterson, X. Dong, P. Khargonekar, V. Nair, and D. Grimard, "Methodology for feedback variable selection for control of semiconductor manufacturing processes-Part 2: Application to reactive ion etching," IEEE Trans. Semicond. Manuf., vol. 16, no. 4, pp. 588-597, Nov. 2003.

[89] O. D. Patterson and P. P. Khargonekar, "Reduction of loading effect in reactive ion etching using real-time closed-loop control," J. Electrochem. Soc., vol. 144, no. 8, pp. 2865-2871, 1997.

[90] B. Rashap, M. Elta, H. Etemad, J. Fournier, J. Freudenberg, M. Giles, J. Grizzle, P. Kabamba, P. Khargonekar, S. Lafortune, J. Moyne, D. Teneketzis, and F. Terry, "Control of semiconductor manufacturing equipment: Real-time feedback control of a reactive ion etcher," IEEE Trans. Semicond. Manuf., vol. 8, no. 3, pp. 286-297, Aug. 1995.

[91] M. Hankinson, T. Vincent, K. Irani, and P. Khargonekar, "Integrated real-time and run-to-run control of etch depth in reactive ion etching," IEEE Trans. Semicond. Manuf., vol. 10, no. 1, pp. 121-130, Feb. 1997.

[92] M. Hankinson, T. L. Vincent, K. Irani, and P. P. Khargonekar, "Combined real-time and run-to-run control of etch depth and spatial uniformity in plasma etching," J. Electrochem. Soc., vol. 144, no. 7, pp. 2473-2479, 1997.
[93] H. M. Park, C. Garvin, D. S. Grimard, and J. W. Grizzle, "Control of ion energy in a capacitively coupled reactive ion etcher," J. Electrochem. Soc., vol. 145, no. 12, pp. 4247-4252, 1998.

[94] C. Lin, K.-C. Leou, and K.-M. Shiao, "Feedback control of chlorine inductively coupled plasma etch processing," J. Vac. Sci. Technol., vol. 23, pp. 281-287, 2005.

[95] V. Milosavljevic, A. Ellingboe, C. Gaman, and J. Ringwood, "Real time plasma control in a dual frequency confined plasma etcher," $J$. Appl. Phys., 2008, vol. Accepted.

[96] C. Lin, K.-C. Leou, H.-M. Huang, and C.-H. Hsieh, "Feedback control of plasma electron density and ion energy in an inductively coupled plasma etcher," J. Vac. Sci. Technol. A, Vac. Surf. Films, vol. 27, no. 1, pp. 157-164, 2009.

[97] C. Hanish, J. Grizzle, J. Teny, and F. L. , "Estimating and controlling atomic chlorine concentration via actinometry," IEEE Trans. Semicond. Manuf., vol. 12, no. 3, pp. 323-331, Aug. 1999.

[98] P. I. Klimecky, J. W. Grizzle, and F. L. Terry, "Compensation for transient chamber wall condition using real-time plasma density feedback control in an inductively coupled plasma etcher," J. Vac. Sci. Technol. A, vol. 21, no. 3, pp. 706-717, 2003.

[99] T. L. Vincent, P. P. Khargonekar, and F. L. Terry, Jr., "End point and etch rate control using dual-wavelength laser reflectometry with a nonlinear estimator," J. Electrochem. Soc., vol. 144, no. 7, pp. 2467-2472, 1997.

[100] D. Stokes and G. May, "Real-time plasma etch control using in-situ sensors and neural networks," in Proc. IEEE Int. Conf. Contr. App., 1999, vol. 1, pp. 779-783.

[101] A. Armaou, J. Baker, and P. D. Christofides, "Feedback control of plasma etching reactors for improved etching uniformity," Chem. Eng. Sci., vol. 56, no. 4, pp. 1467-1475, 2001.

[102] D. Stokes and G. May, "Indirect adaptive control of reactive ion etching using neural networks," IEEE Trans. Robot. Autom., vol. 17, no. 5, pp. 650-657, Oct. 2001.

[103] I. Rosen, T. Parent, B. Fidan, C. Wang, and A. Madhukar, "Design, development, and testing of real-time feedback controllers for semiconductor etching processes using in situ spectroscopic ellipsometry sensing," IEEE Trans. Contr. Syst. Technol., vol. 10, no. 1, pp. 64-75, Jan. 2002.

[104] D. Sung, S. Jeong, Y. Park, V. N. Volynets, A. G. Ushakov, and G.-H. Kim, "Effect on plasma and etch-rate uniformity of controlled phase shift between rf voltages applied to powered electrodes in a triode capacitively coupled plasma reactor," J. Vac. Sci. Technol. A, Vac. Surf. Films, vol. 27, no. 1, pp. 13-19, 2009.

[105] J. Moyne, E. D. Castillo, and A. M. Hurwitz, Run-to-Run Control in Semiconductor Manufacturing. Boca Raton, FL: CRC Press, 2001.

[106] S. Butler and J. Stefani, "Supervisory run-to-run control of polysilicon gate etch using in situ ellipsometry," IEEE Trans. Semicond. Manuf., vol. 7, no. 2, pp. 193-201, May 1994.

[107] A. dePaor, "Some new thoughts on dead-beat control in sampled-data systems," IEEE Trans. Instrum. Meas., vol. 12, no. 2, pp. 91-96, Feb. 1990.

[108] Y. Zheng, Q.-H. Lin, D. S.-H. Wang, S.-S. Jang, and K. Hui, "Stability and performance analysis of mixed product run-to-run control," J. Process Contr., vol. 16, no. 5, pp. 431-443, 2006.

[109] M.-D. Ma, C.-C. Chang, S.-S. Jang, and D. S.-H. Wong, "Mixed product run-to-run process control-An ANOVA model with ARIMA disturbance approach," J. Process Contr., vol. 19, no. 4, pp. 604-614, 2009.

[110] J. Wang and Q. P. He, "A bayesian approach for disturbance detection and classification and its application to state estimation in run-to-run control," IEEE Trans. Semicond. Manuf., vol. 20, no. 2, pp. 126-136, May 2007.

[111] E. Del Castillo and J.-Y. Yeh, "An adaptive run-to-run optimizing controller for linear and nonlinear semiconductor processes," IEEE Trans. Semicond. Manuf., vol. 11, no. 2, pp. 285-295, May 1998.

[112] C. H. Jen, B. C. Jiang, and S. K. S. Fan, "General run-to-run (R2R) control framework using self-tuning control for multiple-input multiple-output (MIMO) processes," Int. J. Prod. Res., vol. 42, no. 20, pp. 4249-4270, 2004.

[113] S.-T. Tseng, W. Song, and Y.-C. Chang, "An Initial Intercept Iteratively Adjusted (IIIA) controller: An enhanced double EWMA feedback control scheme," IEEE Trans. Semicond. Manuf., vol. 18, no. 3, pp. 448-457, Aug. 2005.

[114] J. Wang, Q. He, S. Qin, C. Bode, and M. Purdy, "Recursive least squares estimation for run-to-run control with metrology delay and its application to STI etch process," IEEE Trans. Semicond. Manuf., vol. 18, no. 2, pp. 309-319, May 2005.

[115] S.-K. S. Fan and Y. Lin, "Multiple-input dual-output adjustment scheme for semiconductor manufacturing processes using a dynamic dual-response approach," Eur. J. Oper. Res., vol. 180, no. 2, pp. 868-884, 2007. 
[116] S. Firth, W. Campbell, A. Toprac, and T. Edgar, "Just-in-time adaptive disturbance estimation for run-to-run control of semiconductor processes," IEEE Trans. Semicond. Manuf., vol. 19, no. 3, pp. 298-315, Aug. 2006.

[117] E. Palmer, W. Ren, and C. J. Spanos, "Control of photoresist properties: A Kalman filter based approach," IEEE Trans. Semicond. Manuf., vol. 9, no. 2, pp. 208-214, May 1996.

[118] A. Chen and R.-S. Guo, "Age-based double EWMA controller and its application to cmp processes," IEEE Trans. Semicond. Manuf., vol. 14, no. 1, pp. 11-19, Feb. 2001.

[119] E. Del Castillo and R. Rajagopal, "A multivariate double EWMA process adjustment scheme for drifting processes," IIE Trans., vol. 34, no. 12 , pp. 1055-1068, 2002.

[120] D. Boning, W. Moyne, T. Smith, J. Moyne, and A. Hurwitz, "Practical issues in run by run process control," in Proc. IEEE/SEMI Adv. Semicond. Manuf. Conf., 1995, pp. 201-208.

[121] S.-T. Tseng, R.-J. Chou, and S.-P. Lee, "A study on a multivariate EWMA controller," IIE Trans., vol. 34, no. 6, pp. 541-549, 2002.

[122] E. D. Castillo, "Long run and transient analysis of a double EWMA feedback controller," IIE Trans., vol. 31, no. 12, pp. 1157-1169, 1999.

[123] J. Card, "A study in dynamic neural control of semiconductor fabrication processes," IEEE Trans. Semicond. Manuf., vol. 13, no. 3, pp. 359-365, May 2000.

[124] J. Baras and N. Patel, "A framework for robust run by run control with lot delayed measurements," IEEE Trans. Semicond. Manuf., vol. 10, no. 1, pp. 75-83, Feb. 1997.

[125] T. Smith and D. Boning, "A self-tuning EWMA controller utilizing artificial neural network function approximation techniques," IEEE Trans. Comp., Packag., Manuf. Technol., vol. 20, no. 2, pp. 121-132, Jun. 1997.

[126] D. Boning, W. Moyne, T. Smith, J. Moyne, R. Telfeyan, A. Hurwitz, S. Shellman, and J. Tayor, "Run by run control of chemical-mechanical polishing," IEEE Trans. Compon., Packag., Manuf. Technol., vol. 19, no. 4, pp. 307-314, Dec. 1996.

[127] J. Chen and F. Wang, "PLS based dEWMA run-to-run controller for MIMO non-square semiconductor processes," J. Process Contr., vol. 17, no. 4, pp. 309-319, 2007.

[128] R. Rajagopal and E. Del Castillo, "An analysis and MIMO extension of a double EWMA run-to-run controller for non-squared systems," Int. J. Reliab., Quality Safety Eng. (IJRQSE), vol. 10, no. 4, pp. 417-428, 2003.

[129] C. Zhang, H. Deng, and J. S. Baras, "Run-to-run control methods based on the DHOBE algorithm," Automatica, vol. 39, no. 1, pp. 35-45, 2003.

[130] S. Ramaswamy and J. Lee, "Robust forecasts and run-to-run control for processes with linear drifts," in Proc. Amer. Contr. Conf., Denver, CO, Jun. 2003, vol. 5, pp. 3986-3991.

[131] J. Card, D. Sniderman, and C. Klimasauskas, "Dynamic neural control for a plasma etch process," IEEE Trans. Neural Netw., vol. 8, no. 4, pp. 883-901, Jul. 1997.

[132] J. Card, M. Naimo, and W. Ziminsky, "Run-to-run process control of a plasma etch process with neural network modelling," Quality Reliab. Eng. Int., vol. 14, pp. 247-260, 1998.

[133] S. Limanond, J. Si, and Y.-L. Tseng, "Production data based optimal etch time control design for a reactive ion etching process," IEEE Trans. Semicond. Manuf., vol. 12, no. 1, pp. 139-147, Feb. 1999.

[134] X. Wang and R. Mahajan, "Artificial neural network model-based run-to-run process controller," IEEE Trans. Compon., Packag., Manuf. Technol., vol. 19, no. 1, pp. 19-26, Jan. 1996.

[135] M. Nagase and K. Tokashiki, "Advanced gate etching for accurate CD control for 130-nm node ASIC manufacturing," IEEE Trans. Semicond. Manuf., vol. 17, no. 3, pp. 281-285, Aug. 2004.

[136] K. Miwa, T. Inokuchi, T. Takahashi, A. Oikawa, and K. Imaoka, "Reduction of depth variation in an Si etching process by applying an optimized run-to-run control system," IEEE Trans. Semicond. Manuf., vol. 18, no. 4, pp. 517-521, Nov. 2005.

[137] J. Davis, P. Mozumder, R. Burch, C. Fernando, P. Apte, S. Saxena, S. Rao, and K. Vasanth, "Automatic synthesis of equipment recipes from specified wafer-state transitions," IEEE Trans. Semicond. Manuf., vol. 11, no. 4, pp. 527-536, Nov. 1998.

[138] J. R. Moyne, N. Chaudhry, and R. Telfeyan, "Adaptive extensions to a multibranch run-to-run controller for plasma etching," in Proc. 41st Nat. Symp. Amer. Vac. Soc., 1995, vol. 13, pp. 1787-1791.

[139] T.-B. Koh, S.-Y. Cha, K. B. Woo, D.-S. Moon, K. H. Kwak, and H. S. Chang, "Development of integrated process control system utilizing neural network for plasma etching," in Proc. 17th IEEE/CPMT Int. Electron. Manuf. Technol. Symp., 1995, pp. 218-223.
[140] E. Rietman and R. Frye, "A genetic algorithm for low variance control in semiconductor device manufacturing: Some early results," IEEE Trans. Semicond. Manuf., vol. 9, no. 2, pp. 223-229, May 1996.

[141] R. Ganesan, T. Das, and K. Ramachandran, "A multiresolution analysis-assisted reinforcement learning approach to run-by-run control," IEEE Trans. Robot. Autom., vol. 4, no. 2, pp. 182-193, Apr. 2007.

[142] T. Edgar, W. Campbell, and C. Bode, "Model-based control in microelectronics manufacturing," in Proc. 38th IEEE Conf. Decision Contr., 1999, vol. 4, pp. 4185-4191.

[143] W. Campbell, S. Firth, A. Toprac, and T. Edgar, "A comparison of run-to-run control algorithms," in Proc. Amer. Contr. Conf., 2002, vol. 3, pp. 2150-2155.

[144] E. Hamby, P. Kabamba, and P. Khargonekar, "A probabilistic approach to run-to-run control," IEEE Trans. Semicond. Manuf., vol. 11, no. 4, pp. 654-669, Nov. 1998.

[145] L. Westphal, Handbook of Control Systems Engineering. New York: Springer, 2001.

[146] W. E. Levine, The Control Handbook. Boca Raton, FL: CRC Press, 2009.

[147] S. Qin and T. Badgewell, "An overview of industrial model predictive control technology," in AIChe Symp. Chem. Process Contr. V, 1997, vol. 93 , no. 316 , pp. 232-256.

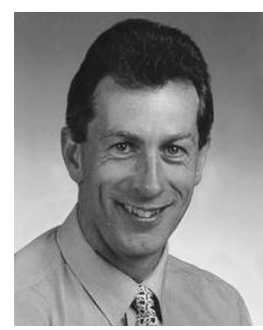

John V. Ringwood (M'87-SM'97) received the Diploma in electrical engineering from Dublin Institute of Technology, Ireland, in 1981 and the $\mathrm{Ph} . \mathrm{D}$. degree in control systems from Strathclyde University, Scotland, U.K., in 1985.

He was with the School of Electronic Engineering, Dublin City University, Ireland, from 1985 to 2000. During that time, he held visiting positions with Massey University and the University of Auckland, New Zealand. He is currently a Professor of electronic engineering with the National University of Ireland (NUI), Maynooth, and Associate Dean for Engineering in the Faculty of Science and Engineering. He was Head of the Electronic Engineering Department at NUI Maynooth from 2000 to 2005, developing the department from a greenfield site. His research interests cover a number of areas, including time-series modelling, wave energy, control of plasma processes, and biomedical engineering.

Prof. Ringwood is a Fellow the Institution of Engineers of Ireland. He is a Chartered Engineer.

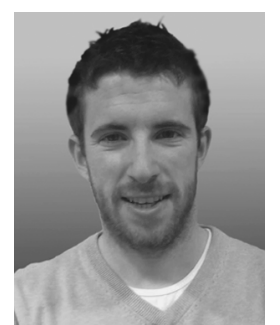

Shane Lynn (S'09) received the bachelor's degree in electrical engineering with computers from the National University of Ireland, Maynooth, in 2006, where he is currently pursuing the Ph.D.

His dissertation is entitled, "Virtual Metrology for Plasma Etch Processes." He has completed internships with the Ad Astra Rocket Company, Costa Rica, where he worked on virtual metrology for a plasma-propelled rocket engine; and Intel Ireland, where he was a Process Engineer. His principal research interests are data analysis, statistical modelling, and control engineering.

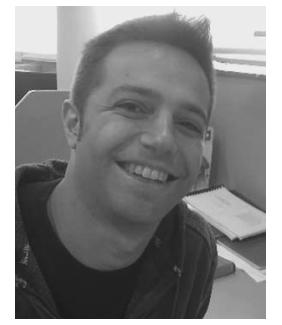

Giorgio Bacelli received the Laurea Magistrale degree in electronic engineering from the Universita' Politecnica delle Marche, Italy, in 2006. He is currently pursuing the Ph.D. degree in the Dynamics and Control Research Group, Electronic Engineering Department, National University of Ireland, Maynooth.

Between 2006 and 2007, he worked on the control of impedance matching for an inductively coupled plasma chamber and on the control of plasma etch processes. Since 2007, he has been working on the modelling and control of ocean wave energy converters for the production of potable water and electricity. 


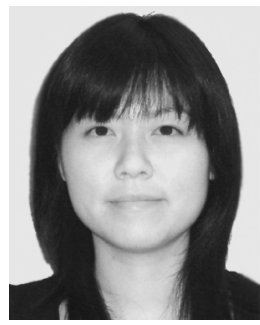

Beibei Ma received the B.Sc. degree from Dalian University of Technology, China, in 2003 and the M.Sc. degree from Dalian Maritime University, China, in 2006. She is currently pursuing the Ph.D. degree in the Electronic Engineering Department, National University of Ireland, Maynooth.

Her research interests include feature extraction, data mining, semiconductor plasma process control, and image processing.

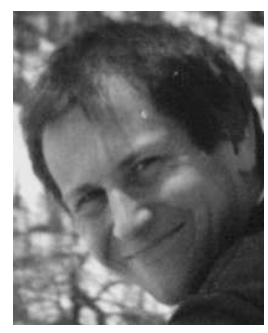

Emanuele Ragnoli received the master's degree in mathematics from Universitá degli Studi di Milano, Italy, in 2002 and the Ph.D. degree in electronic engineering from the Hamilton Institute, National University of Ireland (NUI), Maynooth, in 2006.

He has been with the Electronic Engineering Department, NUI Maynooth, since 2007. His interests cover machine learning, system identification, and control theory applied to semiconductor manufacturing.

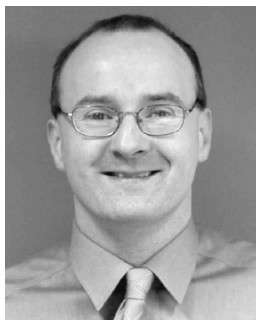

Sean McLoone (S'94-M'96-SM'02) received the M.Eng. degree (with distinction) in electrical and electronic engineering and the Ph.D. degree in control engineering from Queen's University Belfast, Ireland, in 1992 and 1996, respectively.

$\mathrm{He}$ is a Senior Lecturer and Head of the Department of Electronic Engineering, National University of Ireland, Maynooth. His research interests lie in the general area of data-based modelling and analysis of dynamical systems. This encompasses techniques ranging from classical system identification, fault diagnosis, and statistical process control to modern artificial intelligence inspired adaptive learning algorithms and optimization techniques. His research has a strong application focus, with many projects undertaken in collaboration with industry in areas such as process monitoring, control and optimization, time-series prediction, and inline sensor characterization.

Dr. McLoone is a member of Engineers Ireland and the Institution of Engineering and Technology. He is the current Chairman of the UK and Republic of Ireland Section of the IEEE. He is a Chartered Engineer. 